\title{
VIRTUAL VIEWPOINT THREE-DIMENSIONAL PANORAMA
}

\author{
Abdulkadir Iyyaka Audu ${ }^{1}$ and Abdul Hamid Sadka² \\ Department of Electronic and Computer Engineering, Brunel University, London, United \\ Kingdom
}

\begin{abstract}
Conventional panoramic images are known to provide for an enhanced field of view in which the scene always has a fixed appearance. The idea presented in this paper focuses on the use of the concept of virtual viewpoint creation to generate different panoramic images of the same scene with three-dimensional component. Three-dimensional effect in a resultant panorama is realized by superimposing a stereo-pair of panoramic images.
\end{abstract}

\section{KEYWORDS}

Binocular vision, Panoramic images, Cylindrical warping, 3D depth effect, Anaglyph.

\section{INTRODUCTION}

The replication of natural viewing experience derived from television through the addition of depth component has been widely studied [1]. In particular the experience of "immersion" in a three- dimensional (3D) environment has gone through a dynamic growth [2]. In the words of [3], "In face-to-face meetings, we each change our location and direction of gaze so naturally that we hardly give it a thought. In addition, when someone is looking at us, not only do we see that person looking at us, but everyone else can observe that person looking at us from his or her own point of view. It has been shown that mutual gaze enhances human communication". This is depicted in Figure 1.

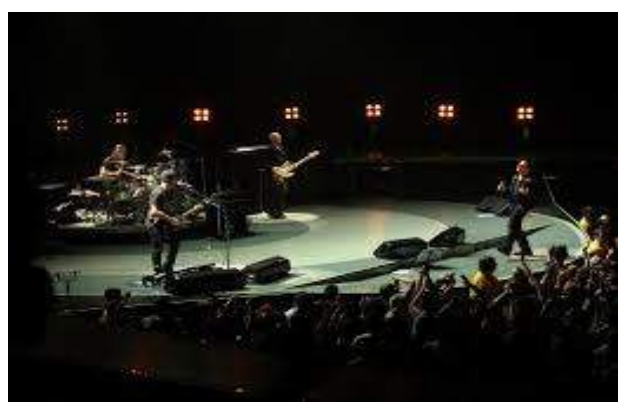

(a)

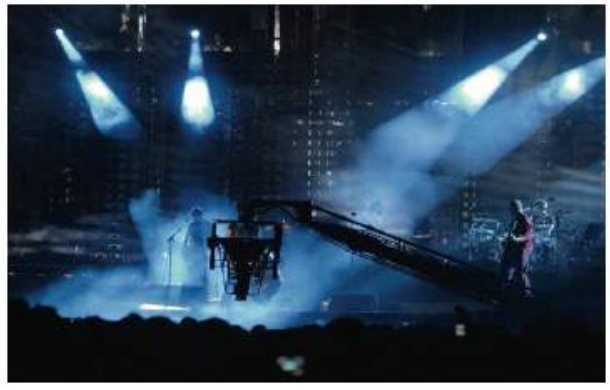

(b)

Figure 1: Examples of live 3D scene depicted. 
The provision of depth perception of images and video in panoramic television is now an active and relevant research topic. The knowledge of stereoscopy is crucial in this regard. It is widely understood that a change of viewpoint with respect to an observed object provides either a slightly or considerably different view of the object. This effect which is attributable to the fact that humans have two eyes and see through perspective projection has been extensively studied in both human vision system (HVS) and machine vision system (MVS). Also, many complex visual tasks, such as reading, detecting camouflaged objects, and eye-hand coordination are also performed more effectively with two eyes than with one, even when the visual display contains no depth [4].

The degree of perceived (3D) realism and enhanced field of view (FOV) are two important factors in vision analysis. In the work of [5], it is observed that retriever of information on the 3D structure and distance of a scene, from a stereo pair of images has become a popular concept in computer vision. In some medical relevant applications robustness, accuracy, and real-time capability are of utmost importance.

A refined analysis has indicated that emerging areas of application in multimedia, with extraordinary standing such as three-dimensional television (3DTV) and free-view television (FVT) are some of the driving factors for this development [6]. Multi-view video is one of the enabling technologies which have recently brought 3DTV and FVT to prominence [7, 8]. In spite of the enormous advantages associated with 3DTV and FVT, [9]. has noted the bandwidth requirement issue, which is critical and challenging for transmitting additional data to render the auxiliary view(s).

Enhanced FOV is the main motivation factor of [10]. It is emphasized that for any FOV enhancement to be achieved, the entire imaging system must have a single effective viewpoint to enable the generation of pure perspective images from a sensed image. The single viewpoint constraint is satisfied by incorporating reflecting mirrors into the conventional imaging system.

In this work, generation of (3D) content from a stereo pair of panoramic views of a scene is proposed. In the view of [11], the following advantages cannot be divorced from stereoscopic view. Depth perception relative to the display surface; spatial localization, allowing concentration on different depth planes; perception of structure in visually complex scenes; improved perception of surface curvature; improved motion judgment; improved perception of surface material type. These benefits give stereoscopic displays improved representation capabilities that allow the user a better understanding or appreciation of the visual information presented.

A panoramic image has the established reputation and capability to provide a 360 degree view of a scene. It is usually obtained by stitching image samples together. It has been widely investigated in the work of $[12,13,14]$. It is also a variant of image-based rendering that allows 3D scenes and objects to be visualized in a realistic way without full 3D model reconstruction. The concept of panoramic image stitching stems from the fundamental deficit in the narrow field of view (FOV) of most compact cameras as depicted in Figure 2. 


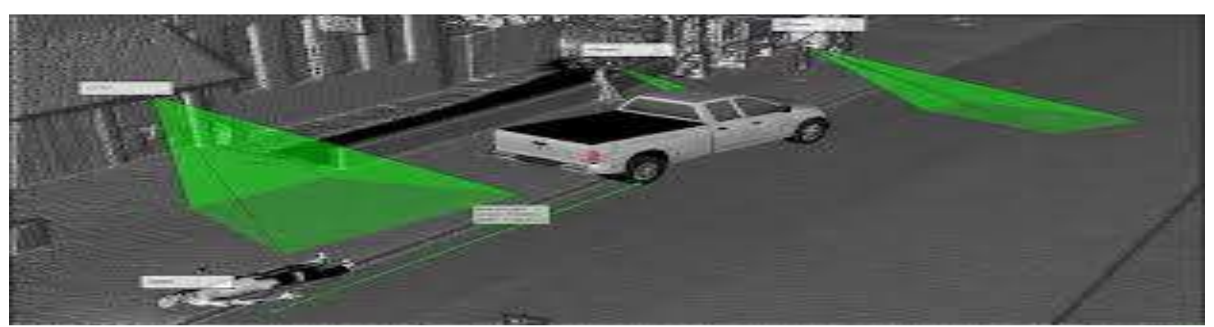

Figure 2: A compact camera image formation process.

The main object of this work is to construct a panoramic image with depth perception. This will be in line with the increasing possibility of panoramic television.

Section II discusses basic concepts of vision, 3D effect, and panoramic view. The focus in section III is the strategy of implementation. Experimental results and discussion are presented in section IV. Conclusion on the proposition is drawn in V.

\section{THREE-DimenSIONAL CONTENT}

\subsection{Binocular Vision and Stereoscopy}

Binocular vision involves the use of two eyes or optical devices for the acquisition of both the optical and geometric property of a scene. It is thought to provide for increased field of view, binocular summation which is the enhancement in the detection of faint objects, and the use of stereoscopic distance or disparity to perceive a scene in 3D and the distance of an object [15]. The amazing effect of significant proportion is the composition of a single image using the single individual image of each eye. This is generally referred to as binocular fusion. The superposition of a pair of images to create depth illusion is known as anaglyph.

In [16], it is believed that parallax, movement parallax, accommodation, convergence, remembered geometry of an object and linear perspective, occlusion, shading, and resolution constitute both physiological and psychological factors, which determine the level of 3D effect we observe as humans. However, parallax and convergence are the most needed factors for anyone to perceive $3 \mathrm{D}$ effect. With accommodation, neurophysiological process varies the radius of curvature of the eye lens to focus the image on the retina. However, with convergence, the continuous movement of the eye ball causes certain angle which decreases with distance to be subtended between the visual axis and optical axis of each eye. This is perhaps linked to the availability of neural algorithm which plays a prominent role in the binarization and manipulation of information the eyes receive.

\subsection{Anaglyph and Synthetic 3D Effect}

At man-made level, the singleness of vision created by neural algorithm in humans is reversed. There are several stereoscopic display methods that can be used to generate $3 \mathrm{D}$ effect. These include lenticular sheet, integral photography, horse blinder barrier, parallax barrier, varifocal mirror, volumetric methods, head mounted display, time sharing method, anaglyph, Brewster's stereoscope, Wheatstone's stereoscope, and 3D movies. From either the projection or interception type of display, one of the two slightly different images of the same object captured with two similar cameras separated by a certain stereoscopic distance is presented to each eye alternately through a filter glass. This concept is demonstrated in Figure 3, [16]. 


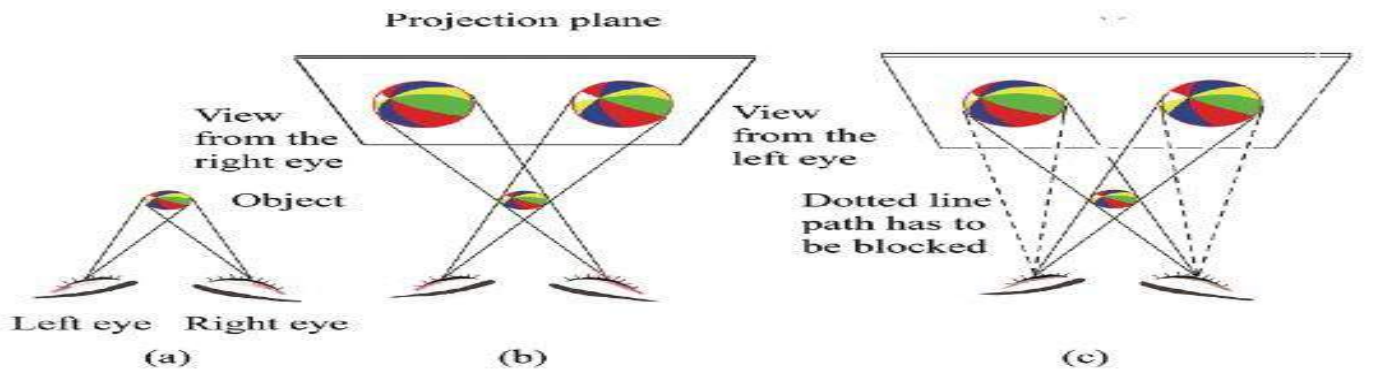

Figure 3: Parallax effect. (a) Object. (b) Projected views of the object. (c) Transposed images.

Whatever display type is used, comfortable view in terms of reduced eye strain or absence of double images from excessive perceived depth is highly required. In [8], it is stated that the mentioned requirement is a function of stereoscopic camera parameters. It is further mentioned that a stereoscopic camera system with parallel axes should be used to avoid the vertical image disparity generated by systems that verge the camera axes. This is because for a parallel camera system, points at infinity have zero disparity and are perceived by the viewer in the plane of the target display. To ensure that corresponding points in the left and right images, at other distances from the viewer, are perceived in the screen plane, the images must be adjusted during or after capture. All these explain the difficulty in producing comfortable images which are often only produced after repeated trial and error. Some common challenges are highlighted in Figure, [17]. 


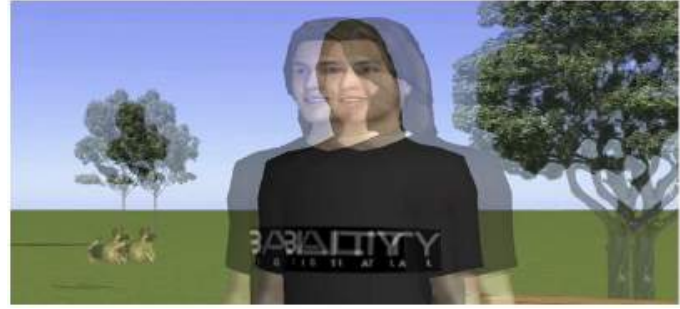

(a)

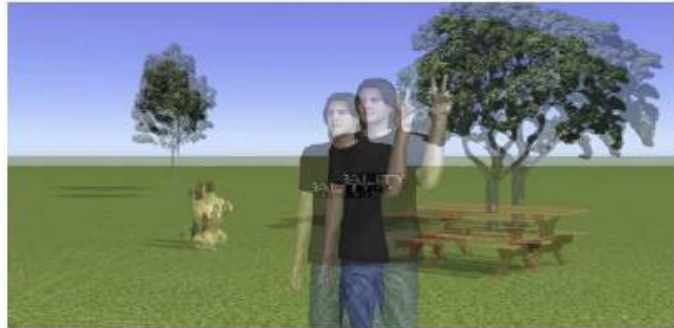

(c)

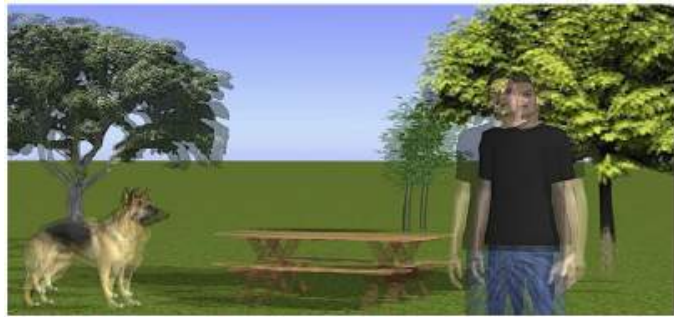

(e)

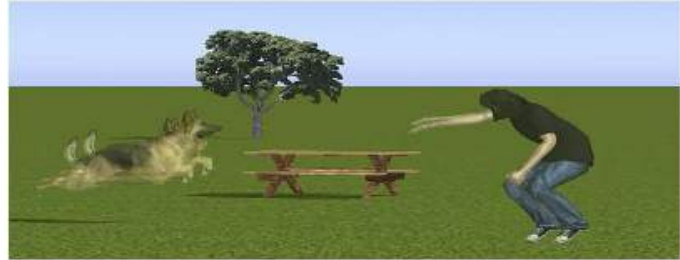

$(\mathrm{g})$

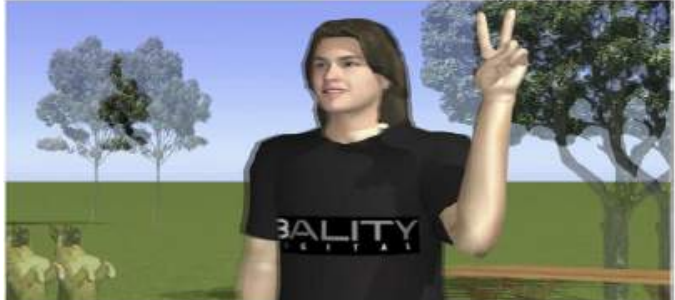

(b)

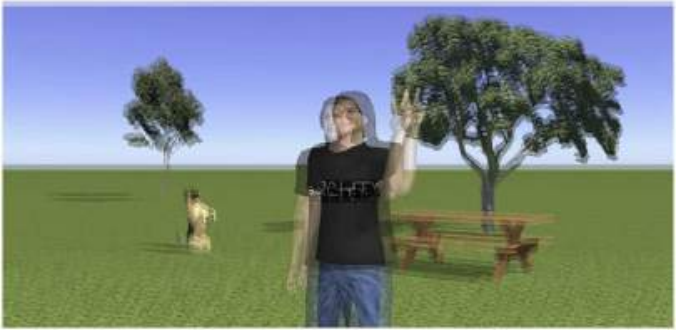

(d)

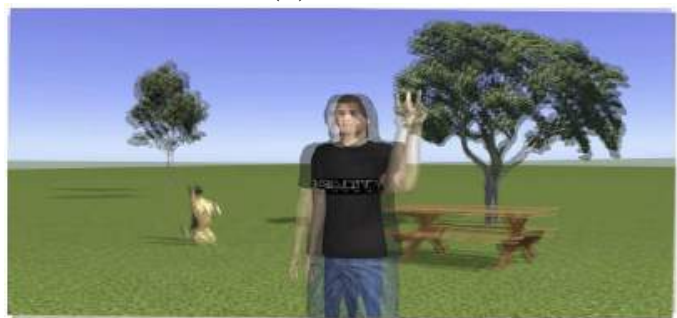

(f)

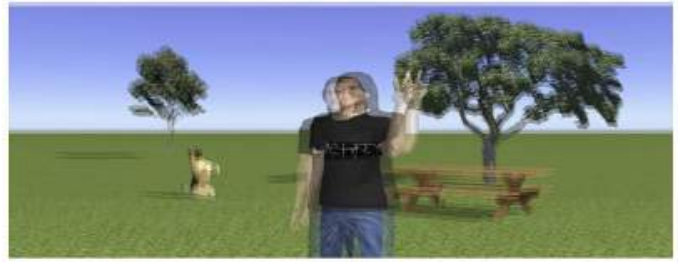

(h)

Figure 4: Causes of 3D discomfort: (a) the distance between the cameras is not adequate, (b) cameras were converged on the wrong point, or one eye was excessively horizontally shifted, (c) lens mismatch, (d) poor manual control on focus distance, and autofocus modes may disagree on the subject distance, (e) keystone appears when the optical axes are not parallel, due to convergence or, less often, strong vertical misalignment, (f) image rotation appears when the camera's optical axis is rotated along the $\mathrm{Z}$ axis, (g) both left and right images are shot without paying great attention to time synchronization, (h) one camera is most likely pointing up or down, or a zoom is off-axis.

\subsection{Anaglyph Computation}

In Figure 5, two similar cameras with a focal length of $\mathrm{f}$ and having a stereoscopic distance of $\mathrm{b}$ between them are used to acquire a world point $(\mathrm{X}, \mathrm{Y}, \mathrm{Z})$. The relationship between the world point and the respective corresponding points $\left(x_{R}, y_{R}\right)$ in the right image and $\left(x_{L}, y_{L}\right)$ in the left is expressed as 
International Journal of Computer Science, Engineering and Information Technology (IJCSEIT), Vol. 4, No.6, December 2014

(1)

$$
\frac{x_{L}}{f}=\frac{X+\frac{b}{2}}{z}, \frac{x_{R}}{f}=\frac{X-\frac{b}{2}}{f}, \frac{y_{L}}{f}=\frac{y_{R}}{f}=\frac{Y}{Z}
$$

The disparity D between corresponding right and left image points is expressed in (2). The reciprocal of $\mathrm{D}$ gives the depth of the world point with respect to the vertical plane contain plane containing the cameras and it decreases with stereoscopic distance. It is also important to note from (1) that disparity is directly proportional to the product of camera focal length and stereoscopic distance, and inversely proportional to the depth D.

$$
D=x_{L}-x_{R}
$$

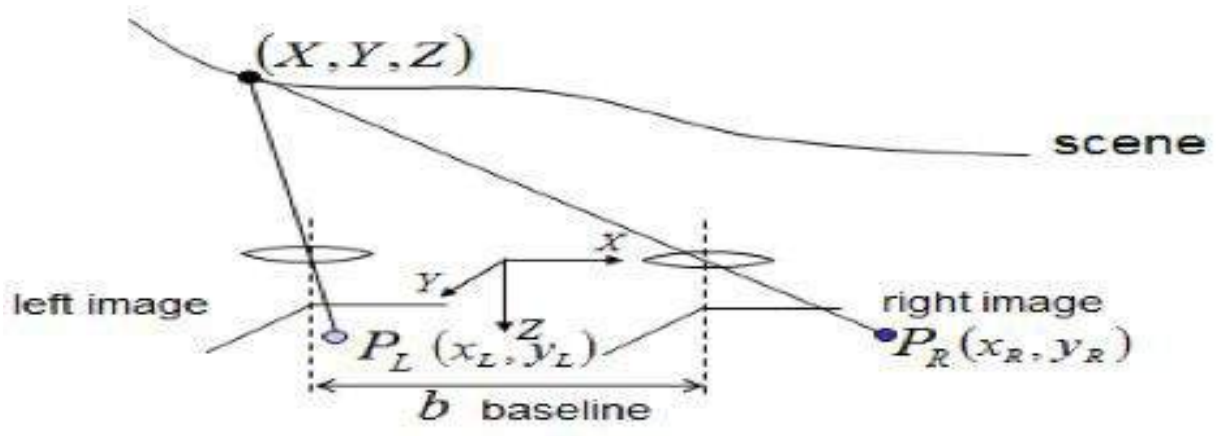

Figure 5: A compact camera image formation process.

It is now been proven that a convincing and comfortable viewing experience can be realized not by maintaining a certain angular disparity as earlier suggested by human factor studies [18] but by compression of scene depth. In [11], this idea is depicted as shown in Figure 6.

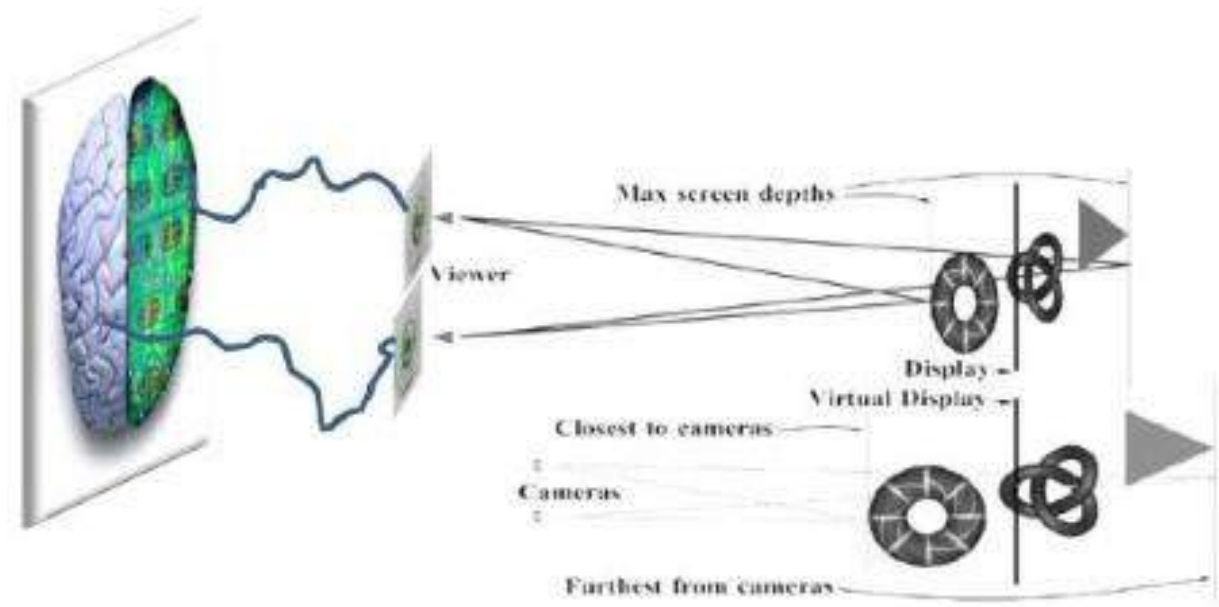

Figure 6: Screen compression: the scene depth (bottom) is compressed (top). 
In the simplified case of a static viewer analyzed in [8], camera separation b can be computed using the relation (3). Where $\mathrm{Z}$ is the distance of the cameras from the 'virtual' display (zeroDisparity-Plane) in the scene, $\mathrm{N}$ is the distance from the cameras to the closest visible points in the scene, $\mathrm{d}_{\mathrm{N}}$ is the disparity, on the display, of objects appearing at the limit $\mathrm{N}$

$$
b=\frac{2 Z^{\prime} \tan \left(\frac{\theta}{2}\right) d_{N} N^{\prime}}{W\left(Z^{\prime}-N^{\prime}\right)+d_{N} N^{\prime}}
$$

The following five types of anaglyph are well known in computer vision. True anaglyphs, colour anaglyphs, grey anaglyphs, half colour anaglyphs, and optimized anaglyphs. According to [19], colour is the general name for all sensations arising from the activity of the retina of the eye and its attached nervous mechanisms, this activity being, in nearly every case in the normal individual, a specific response to radiant energy of certain wavelengths and intensities. This understanding can be explored to seek a mathematical representation of anaglyph. In terms of implementation, colour and grey anaglyphs are usually composed based on the mathematics expressed in (4) and (5) respectively. $A_{r}, A_{g}, A_{b}$ are the colour components of the anaglyph generated from panoramic images 1 and 2 with $\mathrm{r}, \mathrm{g}, \mathrm{b}$ colour components.

$$
\begin{gathered}
{\left[\begin{array}{c}
A_{r} \\
A_{g} \\
A_{b}
\end{array}\right]=\left[\begin{array}{lll}
1 & 0 & 0 \\
0 & 0 & 0 \\
0 & 0 & 0
\end{array}\right]\left[\begin{array}{c}
1_{r} \\
1_{g} \\
1_{b}
\end{array}\right]+\left[\begin{array}{lll}
0 & 0 & 0 \\
0 & 1 & 0 \\
0 & 0 & 1
\end{array}\right]\left[\begin{array}{c}
2_{r} \\
2_{g} \\
2_{b}
\end{array}\right]} \\
{\left[\begin{array}{c}
A_{r} \\
A_{g} \\
A_{b}
\end{array}\right]=\left[\begin{array}{ccc}
0.299 & 0.587 & 0.114 \\
0 & 0 & 0 \\
0 & 0 & 0
\end{array}\right]\left[\begin{array}{c}
1_{r} \\
1_{g} \\
1_{b}
\end{array}\right]+\left[\begin{array}{ccc}
0 & 0 & 0 \\
0.299 & 0.587 & 0.114 \\
0.299 & 0.587 & 0.114
\end{array}\right]\left[\begin{array}{c}
2_{r} \\
2_{g} \\
2_{b}
\end{array}\right]}
\end{gathered}
$$

The limited field of view of conventional imaging devices such as pinhole camera is a problem which is familiar to computer vision researchers and diagnosed by [20, 21]. It is pointed out that while surveillance, teleconferencing, and model acquisition for virtual reality constitute a driving force for an increased field of view, there are several other application areas which are strategically positioned to take advantage of field of view enhancement.

Catadioptric image formation process is widely used for enhancing the field of view of imaging devices. However, image mosaic is favoured in some situations since catadioptric image formation has associated problems of sensor resolution and focusing. Furthermore, in the idea of gradient domain approach presented in [22], of image stitching, the similarity of the sample images and visibility of the seam constitute the cost functions to be optimized. This eventually suppresses both photometric inconsistencies and geometric misalignments between the stitched images.

The method for the generation of panorama falls into two categories namely direct and featurebased methods. It is clear from [23] that accuracy of image registration and closed initialization are the main differences between the two. Feature-based method is considered in this work, since panoramic view generation is one of the image-based rendering methods and features can only be obtained from reference images. 
Of course, the image formation model based on Snell's law developed by [21] is known and well appreciated. In this model, one object point $\mathrm{P} 0$ is traceable to obtain two image coordinates $[u, v]^{T}$ and $\left[u^{\prime}, v^{\prime}\right]^{T}$ on a CCD camera by use of skew ray tracing and taking a single-camera two panoramic views. This camera type is yet to be readily available in the market. In this regard, the use of two separate cameras on a single tripod separated by certain stereoscopic distance is inevitable.

In image mosaicking, the image is first mapped onto the surface of a cylinder, sphere, cube and then the curved surface is unrolled. A method to estimate surface projection is well documented in the work of [14]. Cylindrical warping, [24], can be obtained using either forward or inverse warping as depicted in Figure 7. In forward warping: from image coordinate (x, y), the projected coordinates on the cylinder $\left(x^{\prime}, y^{\prime}\right)$ are given in (6) and (7). Where $S$ is the scaling factor and $f$ equals lens focal length in pixels.

$$
\begin{gathered}
x^{\prime}=S \theta=S \tan ^{-1}\left(\frac{x}{f}\right) \\
y^{\prime}=S h=S\left(\frac{y}{\sqrt{\left(x^{2}+f^{2}\right)}}\right)
\end{gathered}
$$

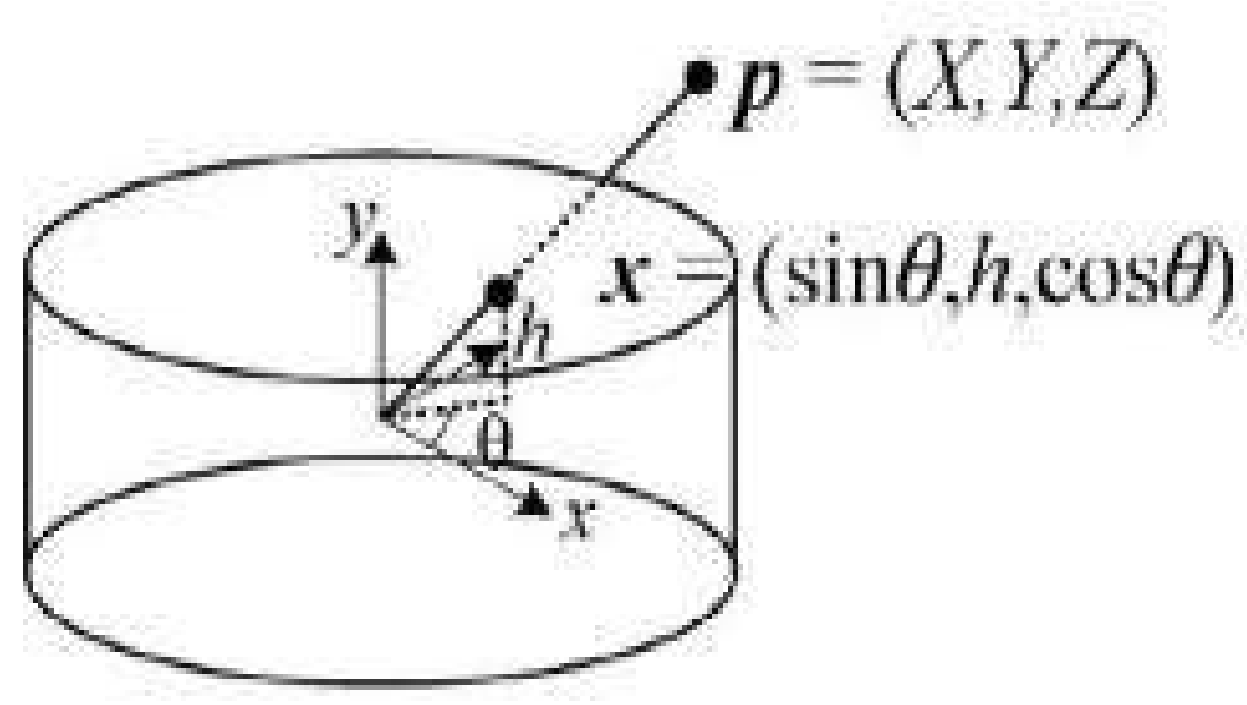

Figure 7: Cylindrical projection.

For inverse warping: inverse mapping from cylindrical coordinates to image $(\mathrm{x}, \mathrm{y})$ is expressed in (8) and (9).

$$
\begin{gathered}
x=f \tan \theta=f \tan \left(\frac{x^{\prime}}{s}\right) \\
y=h \sqrt{\left(x^{2}+f^{2}\right)}=f\left(\frac{y^{\prime}}{s}\right) \sec \left(\frac{x^{\prime}}{s}\right)
\end{gathered}
$$




\section{IMPLEMENTATION STRATEGY}

$$
2014
$$

The implementation of this work is carried out in two stages as shown in Figure 8. First is the generation of two separate panoramic views of a scene. Second is the anaglyph composition.

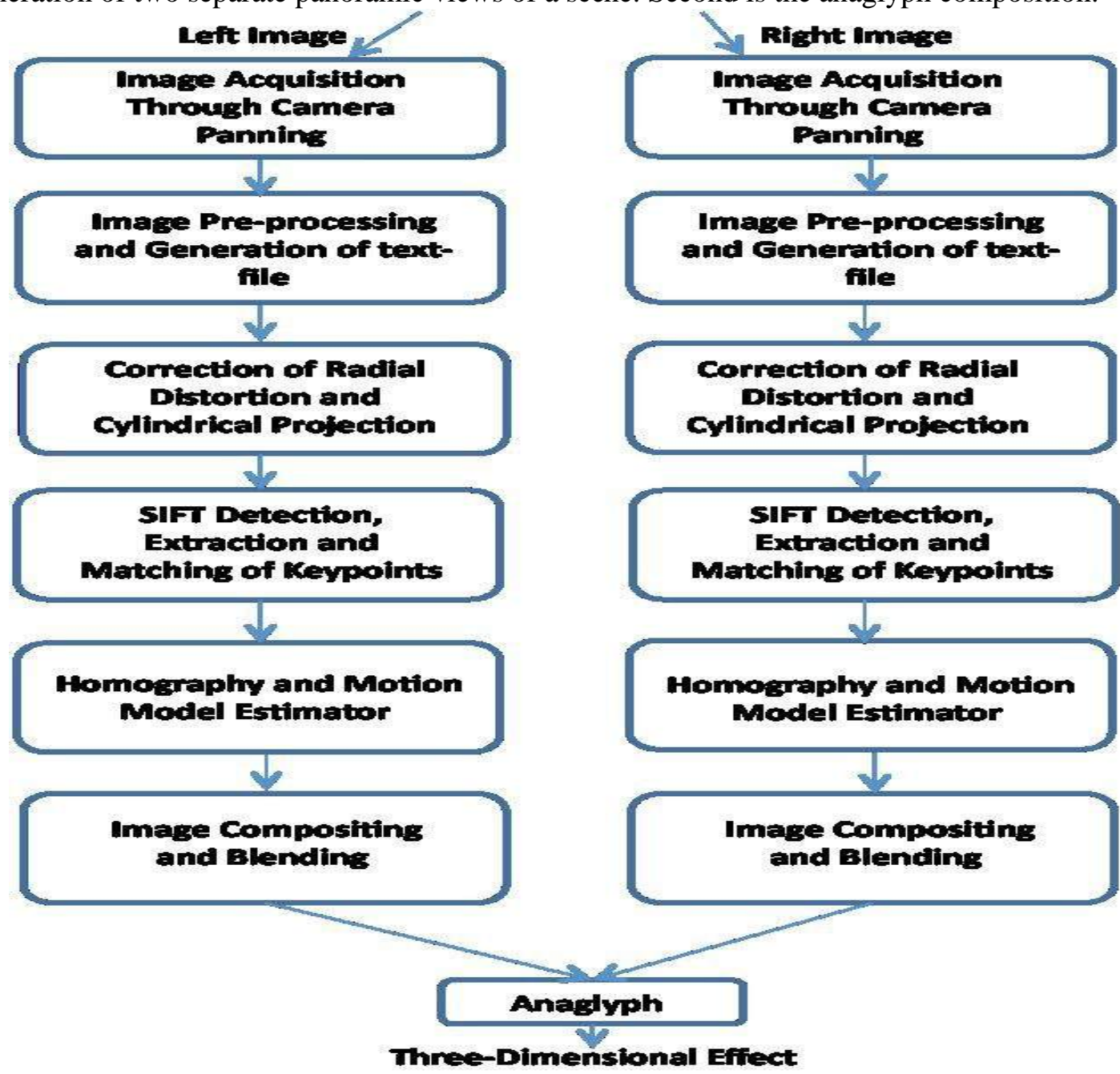

Figure 8: Block diagram for generating 3D effect from two panoramic view.

\subsection{Generation of Panoramic Image}

- The first crucial step in the generation of any panoramic view is the acquisition of image samples of a scene through 360 degrees camera panning. Several images capture different portions of the same scene, with an overlap region viewed in any two images. A path description of each image location is then contained in a text file.

- In this work, a cylinder is used as the projection surface. This allows for an $180 \times 360^{\circ}$ field of view enhancement. This step is then followed by correction of radial distortion associated with image. Two types of radial distortion can be corrected: barrel and 
International Journal of Computer Science, Engineering and Information Technology (IJCSEIT), Vol. 4, No.6, December 2014

pincushion. In "barrel distortion", image magnification decreases with distance from the optical axis. The apparent effect is that of an image which has been mapped around a sphere (or barrel). In pincushion distortion, image magnification increases with the distance from the optical axis. The visible effect is that lines that do not go through the center of the image are bowed inwards, towards the centre of the image, like a pincushion. Brown's (1972) extension of Magill's formulation for variation of radial distortion with focusing still remain potentially attractive. This is in spite of the reverification by $[25,26,27]$, with data of much higher precision than the previous investigations. Brown's distortion. This is expressed in (10) and (11). $\left(\mathrm{x}_{\mathrm{d}}, \mathrm{y}_{\mathrm{d}}\right)$ describes the coordinates of the distorted image while $\left(\mathrm{x}_{\mathrm{u}}, \mathrm{y}_{\mathrm{u}}\right)$ is for the undistorted.

$$
\begin{aligned}
& x_{d}=x_{u}\left(1+\left(k_{1} r^{2}\right)+\left(k_{2} r^{4}\right)\right) \\
& y_{d}=y_{u}\left(1+\left(k_{1} r^{2}\right)+\left(k_{2} r^{4}\right)\right)
\end{aligned}
$$

- Fundamentally, image registration involves the establishment of a motion model which allows for proper integration of useful information from multiple images of the same scene taken at different times, from different viewpoints and/or by different sensors. Depending on the area of application, image registration can be either multi-temporal analysis (different time), multi-view analysis (different viewpoints), scene to model registration (images of a scene and its model are registered), and multimodal analysis (different sensors are used in image acquisition).

- It is well established in literature that irrespective of application area, an image registration is usually implemented under five steps namely: feature detection in which the descriptive image regions called feature points are detected, feature extraction, feature matching, motion model estimation, and image re-sampling and transformation. Scale invariant feature transform (SIFT), [28][20], is used for detection and extraction of a much larger number of features from the images, which reduces the contribution of the errors caused by these local variations in the average error of all feature matching errors. It is characterized by detection and localization of key-points in different scale space images, followed by the assignment of an orientation to each key-point using local image gradients. Then a key-point descriptor is assembled from the local gradient values around each key-point using orientation histograms.

- The accuracy of panoramic mosaic to a large extent is dependent on the image matching technique employed in the establishment of correspondence between one or several images and a reference. Correspondence between any two images is established using feature- based matching. The features common under geometric constraints to the two images called inliers serve as a prerequisite for the computation of projective matrix and subsequently the motion model. The inliers are computed using an algorithm for robust fitting of models in the presence of many data outliers. The composition of the motion model is such that it allows an image to be transformed with respect to another which is considered to be the reference. The obvious consequence of this process is that the transformed image can then be stitched to the reference image at the proper coordinate points.

- Pyramid blending technique is implemented to allow for a smooth transition from one image to the other across the transition boundary. Pyramid blending involves the 
International Journal of Computer Science, Engineering and Information Technology (IJCSEIT), Vol. 4, No.6, December 2014

combination of different frequency bands with different alpha masks. Lower frequencies are mixed over a wide region, and fine details are mixed in a narrow region. This produces gradual transition in lower frequencies, while reducing edge duplications in textured regions.

\subsection{Anaglyph Composition}

The second stage at broad level is the anaglyph composition. Two colour panoramic views left (1) and right (2) are used to construct colour anaglyph.

Trimming adjustment is also used to vary the horizontal disparity until a comfortable and natural looking image is obtained. In the case of colour anaglyph, the RGB components are maintained even after the coding operation.

\section{Simulation RESULTS AND DiSCUSSION}

The results and appropriate discussion about this work is presented as follow. Each image sample is acquired using a compact digital camera at a resolution of $2464 \times 1632$.

\subsection{Results}

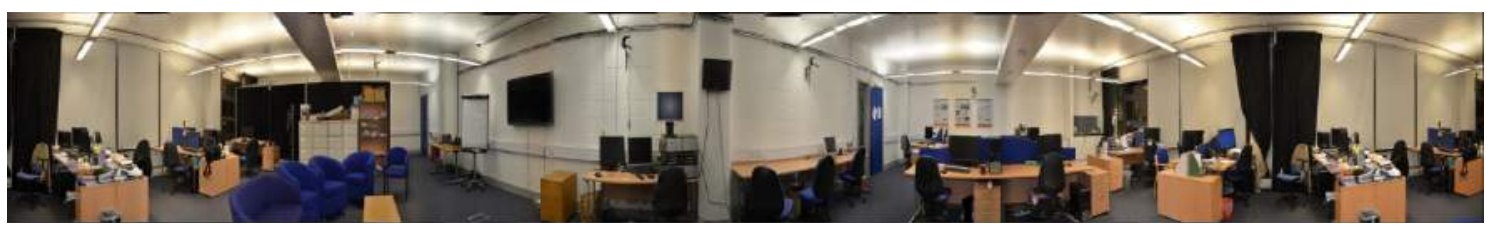

(a)

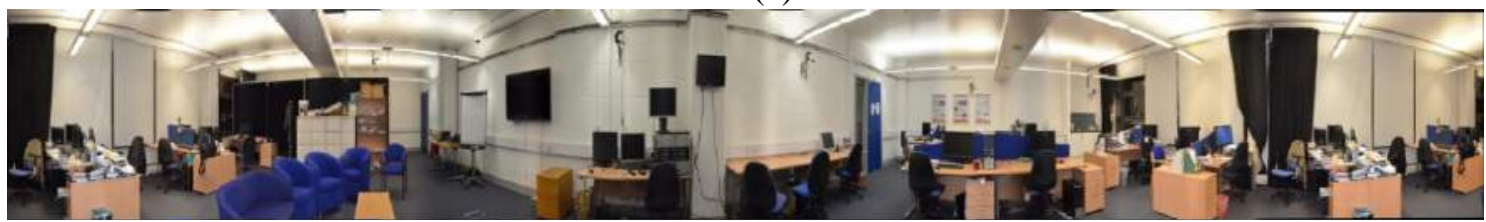

(b)

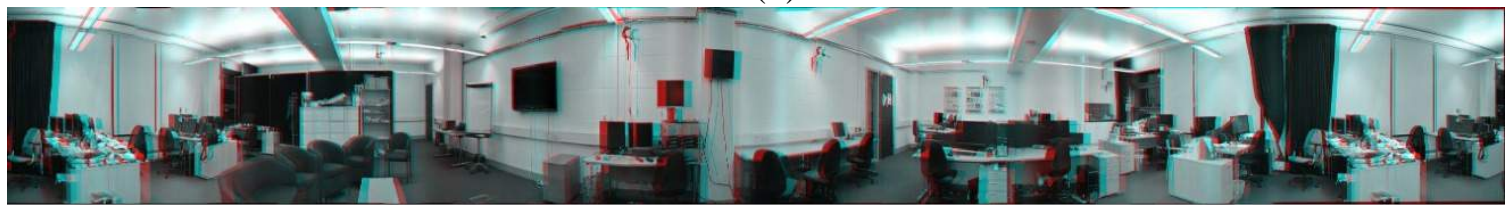

(c)

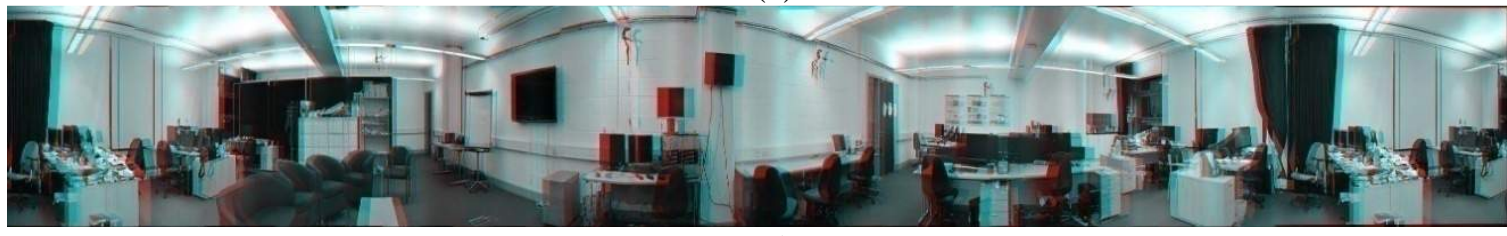

(d) 
International Journal of Computer Science, Engineering and Information Technology (IJCSEIT), Vol. 4, No.6, December

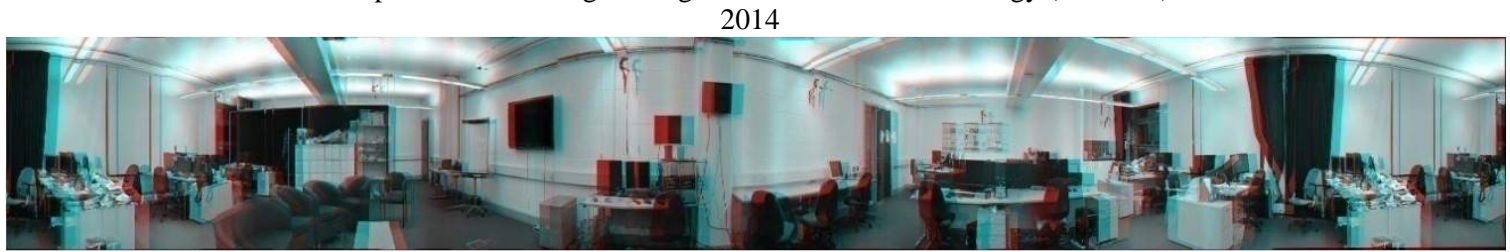

(e)

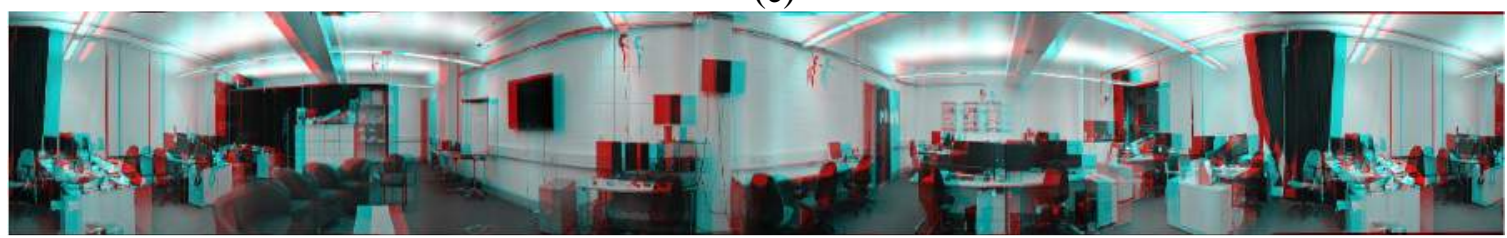

(f)

Figure 9: 3D effect due to stereo panoramic image. (a) and (b) are left and right images. (c), (d), (e), and (f) are images with different 3D effects.

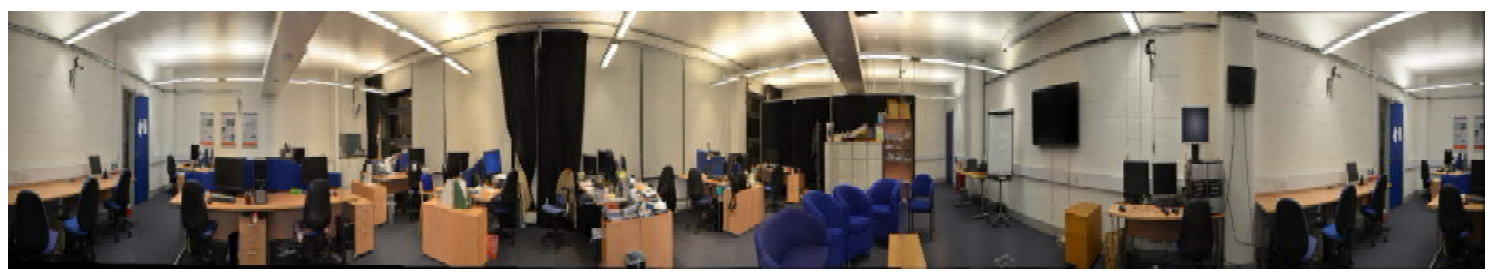

(a)

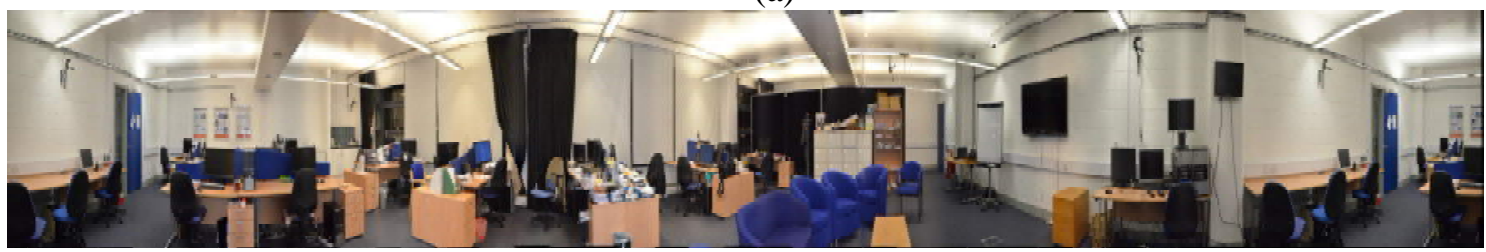

(b)

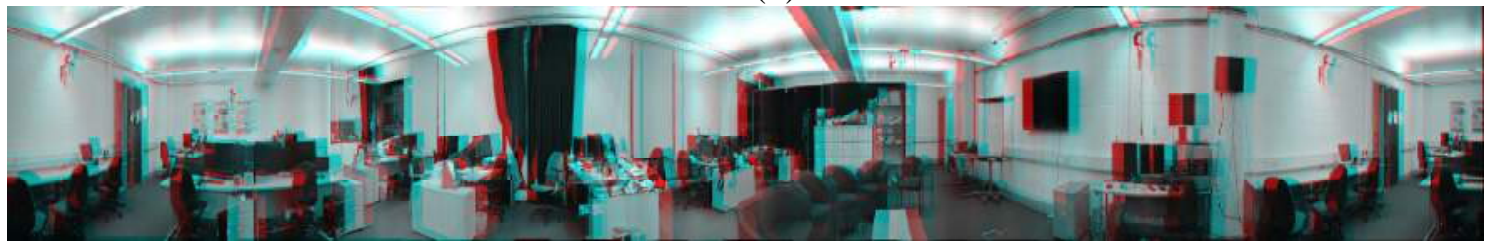

(c)

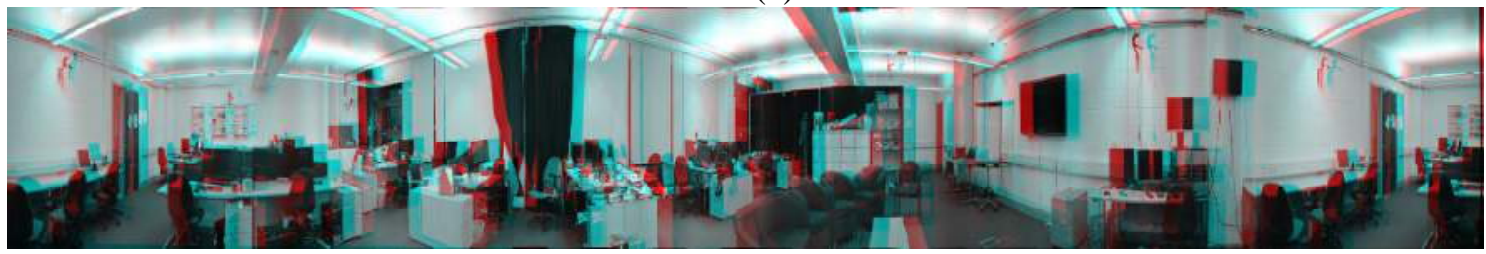

(d)

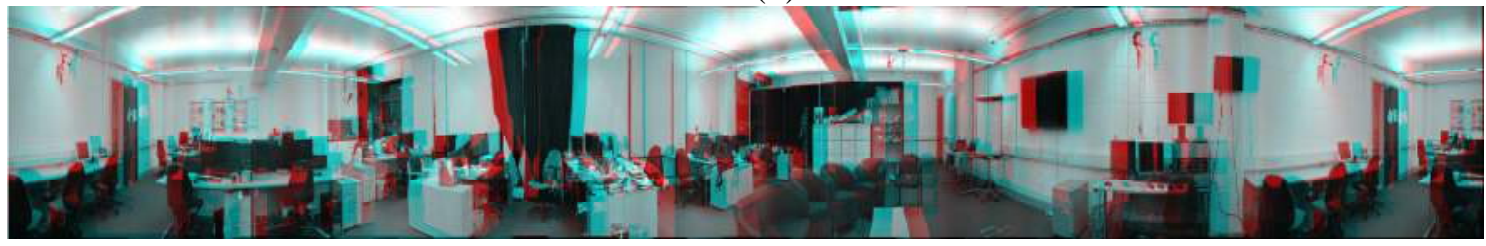


International Journal of Computer Science, Engineering and Information Technology (IJCSEIT), Vol. 4, No.6, December 2014

(e)

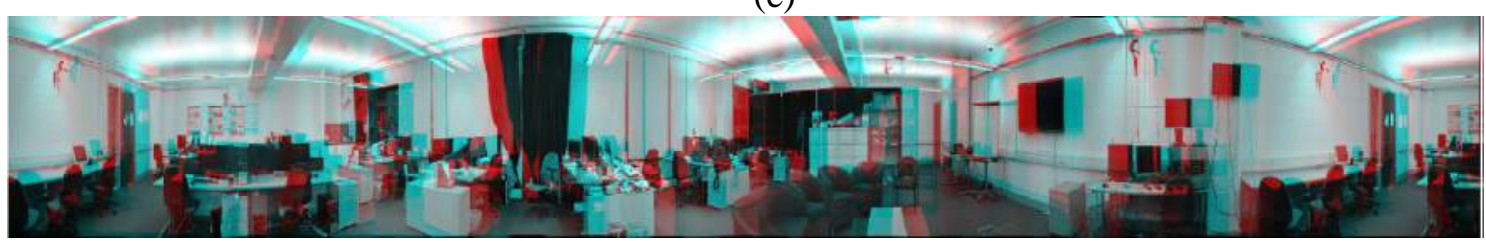

(f)

Figure 10: 3D effect due to stereo panoramic image. (a) and (b) are left and right images. (c),(d), (e), and (f) are images with different 3D effects.

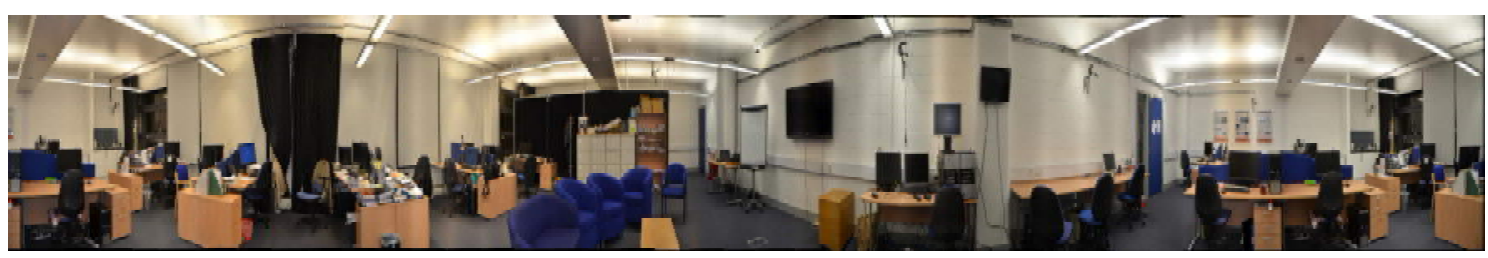

(a)

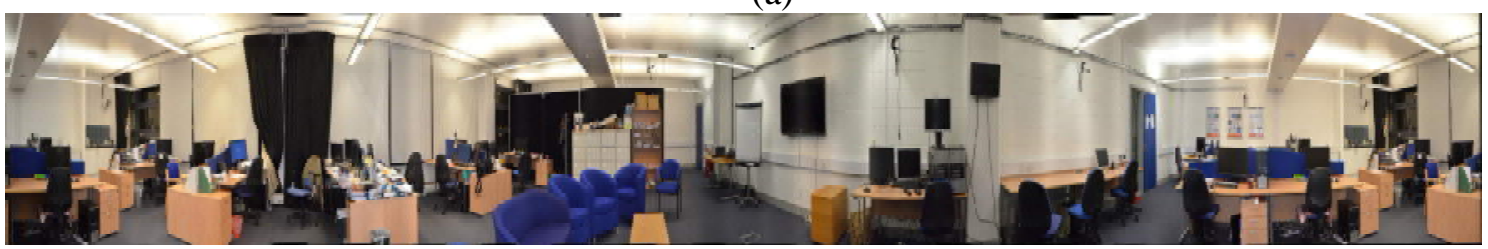

(b)

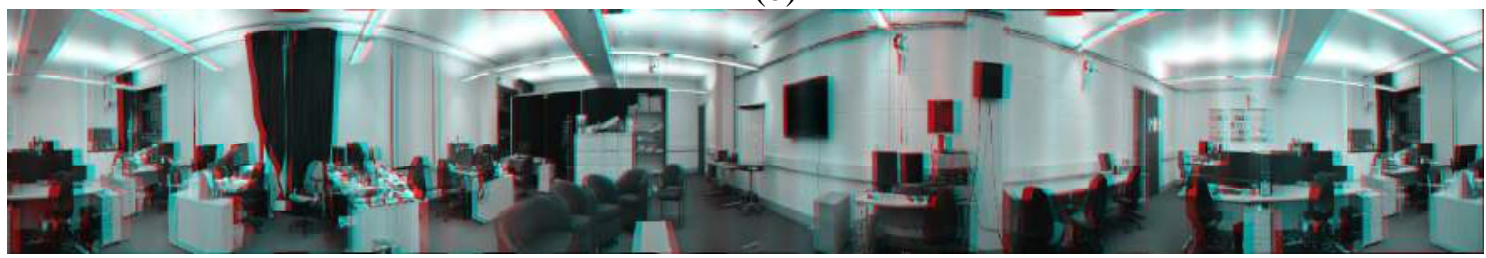

(c)

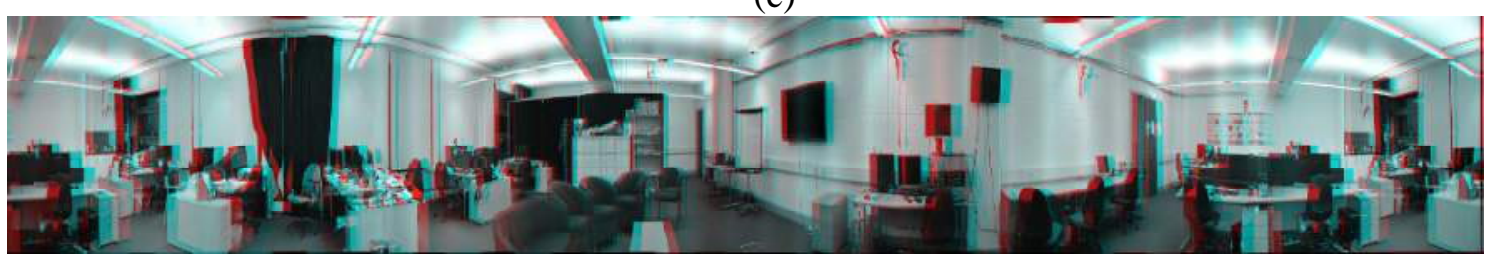

(d)

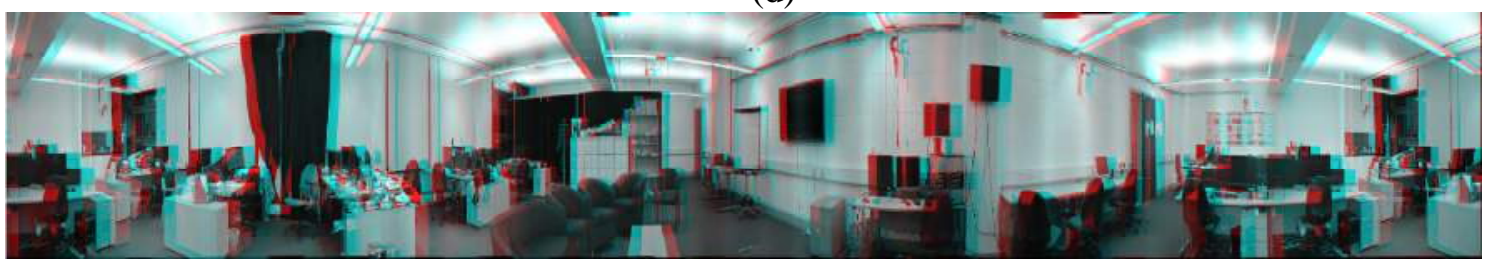

(e) 
International Journal of Computer Science, Engineering and Information Technology (IJCSEIT), Vol. 4, No.6, December

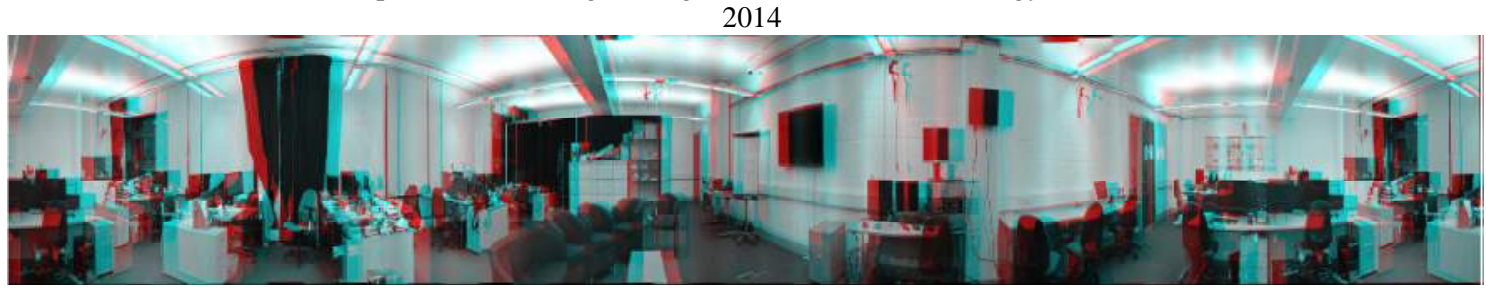

(f)

Figure 11: 3D effect due to stereo panoramic image. (a) and (b) are left and right images. (c),(d), (e), and (f) are images with different 3D effects.

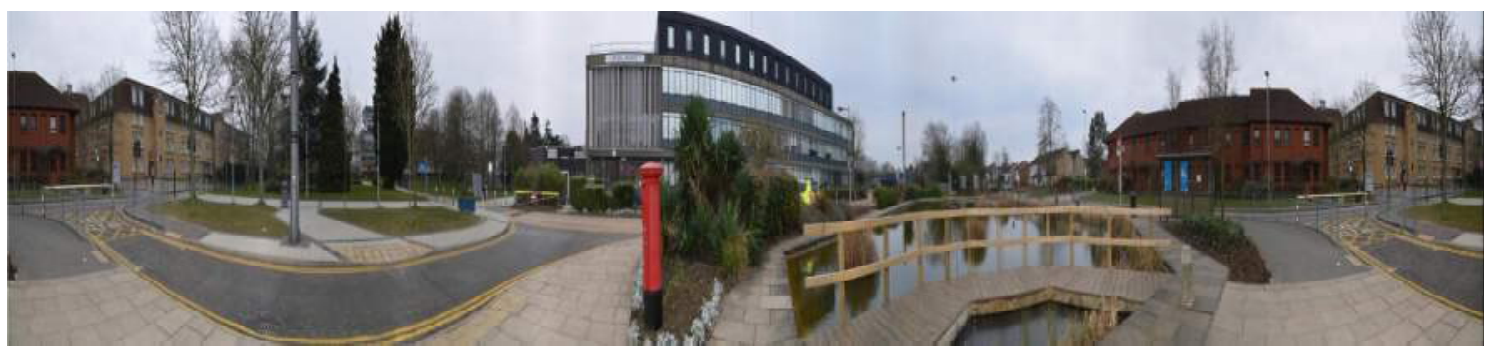

(a)

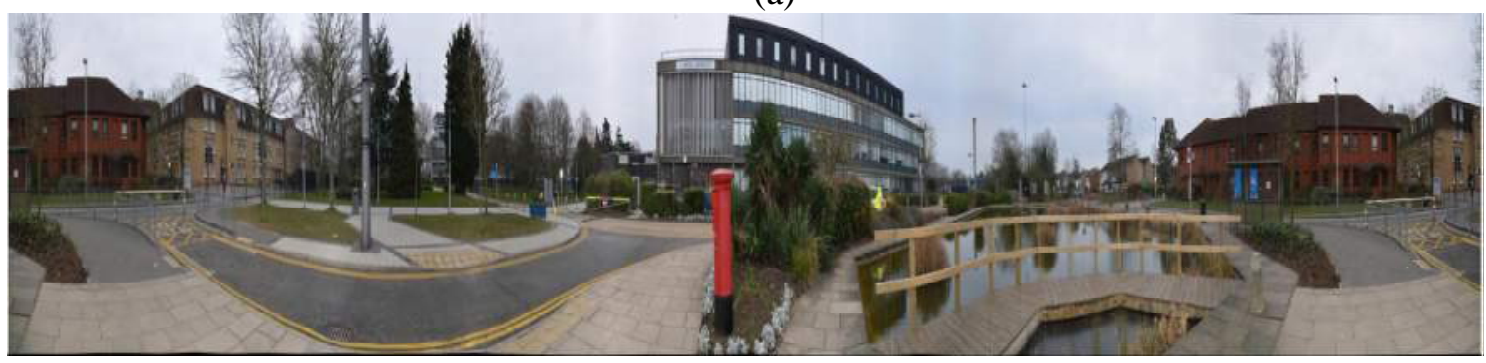

(b)

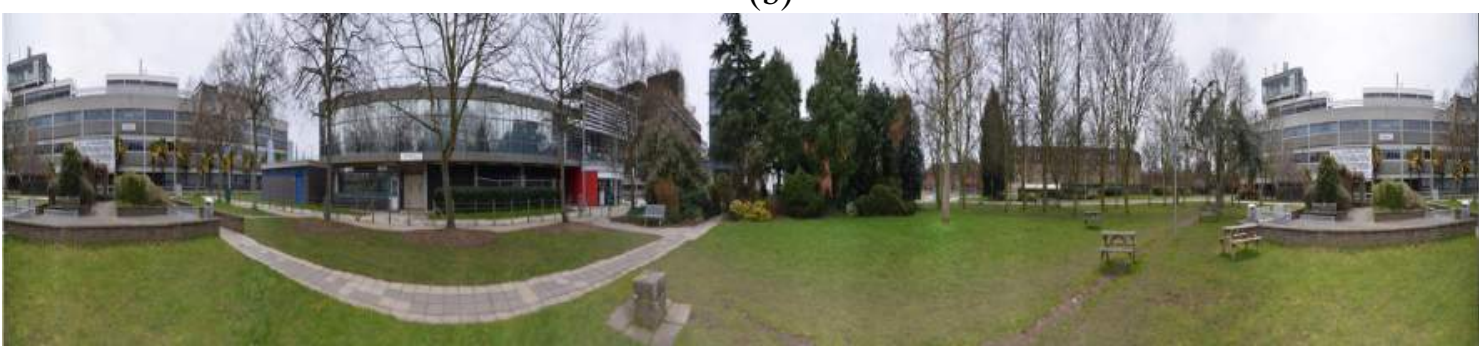

(c)

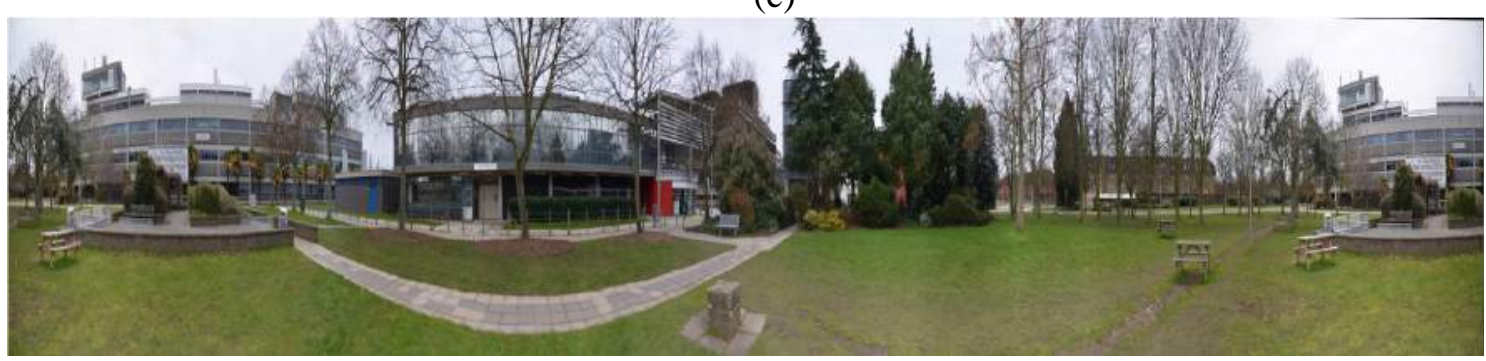

(d) 
International Journal of Computer Science, Engineering and Information Technology (IJCSEIT), Vol. 4, No.6, December 2014

Figure 12: Outdoor scenes: (a) left view and (b) right view of first scene. The second scene also has (c) and (d) as left and right views respectively.

\subsection{Discussion}

All figures, tables, Two sets of image samples at a resolution of $2464 \times 1632$ are acquired using two compact digital cameras (Nikkon D7000) mounted on a single tripod. Each set contains thirty six images. Image acquisition was made with stereoscopic distance of $130 \mathrm{~mm}, 140 \mathrm{~mm}, 150 \mathrm{~mm}$, and $160 \mathrm{~mm}$.

Thirty eight sample images have been used for each of the constructed panoramic views with the first and last images being repeated. One important observation is that, in the panoramic views of Figure 9 through to Figure 11, the observed perspective projection is different for similar objects of the same physical size located at almost the same point in the scene. This is due to change in viewpoint. Also the 3D effect which is observed through a pair of anaglyph glasses increases from (c) through to (f) in the just mentioned figures. What can be noticed is the pop out effect from the screen.

Also two other panoramic image pairs of out-door scenes are presented in Figure 12. 3D effects could equally be generated from each pair.

\section{CONCLUSION}

The generation of 3D effect from two panoramic views whose image samples are obtained from two synchronized cameras, has been demonstrated. Acquisition of image samples is carried out in both indoor and outdoor environments. Little or considerable vegetation has been used as a criteria for the choice of outdoor environment in this work. It is important to note that the quality of 3D effect largely depends on how well the image samples have been well stitched. The subjective assessment based on the viewing experience of a group of people confirms that the resultant depth quality is good and does not cause much eye strain.

\section{REFERENCES}

[1] C. Fehn, E. Cooke, O. Schreer, and P. Kauff, “3d analysis and image-based rendering for immersive tv applications," Signal Processing: Image Communication, vol. 17, no. 9, pp. 705-715, 2002.

[2] A. Mazikowski and J. Lebiedz, "Image projection in immersive 3d visualization laboratory," Proceeding Computer Science, vol. 35, pp. 842-850, 2014.

[3] H. Fuchs, A. State, and J.-C. Bazin, "Immersive 3d telepresence," Computer, vol. 47, no. 7, pp. 4652, 2014.

[4] F. A. Kingdom, "Binocular vision: The eyes add and subtract," Current Biology, vol. 22, no. 1, pp. R22-R24, 2012.

[5] S. Hadjitheophanous, C. Ttofis, A. Georghiades, and T. Theocharides, "Towards hardware stereoscopic 3d reconstruction a real-time fpga computation of the disparity map," in Design, Automation and Test in Europe Conference and Exhibition (DATE), 2010, pp. 1743-1748, IEEE, 2010. 
International Journal of Computer Science, Engineering and Information Technology (IJCSEIT), Vol. 4, No.6, December 2014

[6] S. Bae, H. Lee, H. Park, H. Cho, J. Park, and J. Kim, "The effects of egocentric and allocentric representations on presence and perceived realism: Tested in stereoscopic 3d games," Interacting with Computers, 2012.

[7] S. Chan, H. Y. Shum, and K. T. Ng, "Image-based rendering and synthesis," Signal Processing Magazine, IEEE, vol. 24, no. 6, pp. 22-33, 2007.

[8] C. Lee, Y.-S. Ho, and B. Choi, "Efficient multiview depth video coding using depth synthesis prediction," Optical Engineering, vol. 50, pp. 077004-077004, 07 2011. M3: Article.

[9] C. Gurler, K. Bagci, and A. M. Tekalp, "Adaptive stereoscopic 3d video streaming," in Image Processing (ICIP), 2010 17th IEEE International Conference on, pp. 2409-2412, IEEE, 2010.

[10] S. K. Nayar, "Catadioptric omnidirectional camera," in Computer Vision and Pattern Recognition, 1997. Proceedings, 1997 IEEE Computer Society Conference on, pp. 482-488, IEEE, 1997.

[11] G. R. Jones, D. Lee, N. S. Holliman, and D. Ezra, "Controlling perceived depth in stereoscopic images," in Photonics West 2001-Electronic Imaging, pp. 42-53, International Society for Optics and Photonics, 2001.

[12] L. E. Gurrieri and E. Dubois, "Efficient panoramic sampling of real world environments for imagebased stereoscopic telepresence," in Proc. SPIE 8288, vol. 82882, 2011.

[13] R. Szeliski and H. Y. Shum, "Creating full view panoramic image mosaics and environment maps," in Proceedings of the 24th annual conference on Computer graphics and interactive techniques, pp. 251-258, ACM Press/Addison-Wesley Publishing Co., 1997.

[14] R. Szeliski, "Image alignment and stitching: A tutorial," Foundations and Trends in Computer Graphics and Vision, vol. 2, no. 1, pp. 1-104, 2006.

[15] R. F. van der Willigen, W. M. Harmening, S. Vossen, and H. Wagner, "Disparity sensitivity in man and owl: Psychophysical evidence for equivalent perception of shape-from-stereo," Journal of Vision, vol. 10, no. 1, 2010.

[16] K. Iizuka, Engineering optics, vol. 35. Springer, 2008.

[17] B. Mendiburu, 3d TV and 3d cinema: tools and processes for creative stereoscopy. Taylor and Francis, 2011.

[18] M. Wopking, "Viewing comfort with stereoscopic pictures: An experimental study on the subjective effects of disparity magnitude and depth of focus," Journal of the Society for Information Display, vol. 3, no. 3, pp. 101-103, 1995.

[19] P. Corke, Robotics, Vision and Control: Fundamental Algorithms in MATLAB, vol. 73. Springer, 2011.

[20] S. Baker and S. K. Nayar, "A theory of catadioptric image formation," in Computer Vision, 1998. Sixth International Conference on, pp. 35-42, IEEE, 1998.

[21] C.-K. Sung and C.-H. Lu, "Single-camera panoramic stereo system model based on skew ray tracing," Optik - International Journal for Light and Electron Optics, vol. 123, pp. 594-603, 042012. M3: Article.

[22] A. Levin, A. Zomet, S. Peleg, and Y.Weiss, "Seamless image stitching in the gradient domain," Computer Vision-ECCV 2004, pp. 377-389, 2004.

[23] M. Brown and D. G. Lowe, "Automatic panoramic image stitching using invariant features," International Journal of Computer Vision, vol. 74, no. 1, pp. 59-73, 2007. (document)

[24] E. W. Weisstein, “Cylindrical projection.” From MathWorld-A Wolfram Web Resource. http://mathworld.wolfram.com/CylindricalProjection.html.

[25] J. G. Fryer and D. C. Brown, "Lens distortion for close-range photogrammetry," Photogrammetric Engineering and Remote Sensing, vol. 52, no. 1, pp. 51-58, 1986.

[26] S. Aritan, "Efficiency of non-linear lens distortion models in biomechanical analysis of human movement," Measurement (02632241), vol. 43, pp. 739-746, 07 2010. M3: Article.

[27] M. Goljan and J. Fridrich, "Sensor-fingerprint based identification of images corrected for lens distortion," in Media Watermarking, Security, and Forensics 2012, vol. 8303 The Society of PhotoOptical Instrumentation Engineers (SPIE); Conference code: 88949.

[28] Y. Cui, N. Hasler, T. ThormÃ€hlen, and H. P. Seidel, "Scale invariant feature transform with irregular orientation histogram binning,” Image Analysis and Recognition, pp. 258-267, 2009. 
International Journal of Computer Science, Engineering and Information Technology (IJCSEIT), Vol. 4, No.6, December 2014 